\title{
Internal Jugular Vein Catheterization: The Landmark Technique versus Ultrasonography Guidance in Cardiac Surgery
}

Selcuk Kayir ${ }^{1}$, Sertan Ozyalcin ${ }^{2}$, Guvenc Dogan ${ }^{1}$, Adem Ilkay Diken ${ }^{2}$, Ufuk Turkmen ${ }^{2}$

1. Anesthesiology, Hitit University, Erol Olcok Training and Research Hospital, Corum, TUR 2. Cardiovascular Surgery, Hitit University Erol Olcok Training and Research Hospital, Corum, TUR

$\square$ Corresponding author: Selcuk Kayir, drskayir@gmail.com

\section{Abstract}

\section{Background}

Central venous catheterization is an invasive procedure that must be performed during cardiovascular surgery. The addition of ultrasound guidance to the catheterization technique has shown effectiveness in reducing complications because it allows for the visualization of anatomical variations prior to intervention and the continual visualization of the needle during the placement. The purpose of this study was to evaluate the effectiveness of needle-guiding ultrasound for internal jugular venous cannulation.

\section{Method}

Patients undergoing coronary bypass surgery at Hitit University, department of cardiovascular surgery, from January 2014 to June 2018, were included in the study. The patients were divided into two groups: those with catheterization with ultrasound guidance (Group U) and those with catheterization performed with the anatomic landmark technique (Group L).

\section{Results}

A total of 584 cases were investigated. The success of the procedure and complication rates for both methods were compared. Central vein catheterization with ultrasonography produced success and complication rates significantly better than those for catheterization using the landmark technique ( $\mathrm{p}=0.04$ and $\mathrm{p}=0.00001$, respectively).

\section{Conclusion}

Received 01/23/2019 Review began 01/30/2019 Review ended 02/01/2019 Published 02/07/2019

(C) Copyright 2019

Kayir et al. This is an open access article distributed under the terms of the Creative Commons Attribution License CC-BY 3.0., which permits unrestricted use, distribution, and reproduction in any medium, provided the original author and source are credited.
This study demonstrated that the use of ultrasonography for internal jugular vein catheterization for patients undergoing coronary bypass surgery significantly reduced the complication rates as compared to those of patients where the landmark technique was used for catheterization.

Categories: Anesthesiology, Cardiac/Thoracic/Vascular Surgery

Keywords: cardiac surgery, landmark technique, ultrasonography, central venous catheterization

\section{Introduction}

Central venous catheterization (CVC) is an invasive procedure that must be performed during 
cardiovascular surgery (CVS). Among the indications are hemodynamic monitoring, central venous pressure monitoring, blood transfusion and fluid replacement, a variety of drug infusions, hemodialysis, and hyperalimentation. There may be differences between operators and centers in the routes chosen for CVC. The most commonly chosen intervention route in CVS operations is the internal jugular vein (IJV). The subclavian vein and femoral vein are other anatomic regions where CVC can be performed [1-2]. Complications that may be observed during CVC procedures range from simple local hematomas to complications with mortal progression, including chylothorax, pneumothorax, hemothorax, or mediastinitis.

Until recently, CVC was performed using anatomical indicators (the landmark technique). Although the landmark technique is very standardized, even in the best hands, it has complication rates that cannot be reduced [3]. Currently, this technique is being replaced by methods using imaging modalities as an aid.

CVC guided by ultrasonography (USG) was first described by Legler [4-7]. This method ensures the visualization of anatomic variations before interventions, independent of location and continuous observation of the needle during cannulation. Thus, there are fewer needle passes, shorter catheterization times, and fewer complications. Venous interventions completed with USG are reported to have low complication rates [8].

The aim of this study was to compare the landmark technique versus USG for CVC in patients undergoing CVS in terms of successful intervention numbers, complications developed during and after the procedure, and the mean duration of the procedure.

\section{Materials And Methods}

Following approval from the Hitit University clinical research ethics committee, results from patients undergoing coronary bypass surgery in the department of cardiovascular surgery, from January 2014 to June 2018, were retrospectively examined. A total of 584 patients aged 18-80 years, in the American Society of Anesthesiologists (ASA) I-III, with platelet counts $>50,000$ $\mathrm{mm}^{3}$ and international normalized ratio (INR) $<1.5$ were included in the study. Patients with CVC in the preoperative period for any of a variety of indications, with platelet counts $<50,000$ $\mathrm{mm}^{3}$, INR $>1.5$, infection at the intervention site, valve or aorta surgery (as these patients had different levels of antiaggregant and anticoagulant drug protocols administered in the preoperative period), and planned carotid artery surgery were excluded from the study. The anesthesia forms, surgery notes, intensive care monitoring forms, and hospital database were used to obtain patient data.

Patients in the study were divided into two groups: those with catheterization with USG (Group $\mathrm{U}$ ) and those with catheterization performed with the anatomic landmark technique (Group L). After patients were taken into the operating room for surgery, 5-derivation electrocardiography, pulse oximetry, and arterial pressure monitoring were performed. Patients had anesthesia induction and muscle relaxation, and after intubation, were placed in a slight Trendelenburg position while supine. The head was turned to the side opposite the CVC side. In both groups, local field cleaning was performed with povidone iodine. In Group U, a gel was spread on the USG probe, it was covered with a sterile sheath, and entry was beside the sternocleidomastoid muscle. In Group L, the landmark technique was used to insert the catheter.

The demographic data of the cases were examined. Preoperative hemorrhage profiles, platelet counts, and antiaggregant and anticoagulant intakes were compared. Additionally, the successful venous entry numbers, complications developed during the procedures, and technical successes were evaluated. An unsuccessful procedure was defined as catheterization 
not ensured after the puncture. Complications developed during the procedure were identified as palpable hematoma after the procedure and operation, carotid artery puncture, pneumothorax, or catheter malposition seen on radiological images obtained during or after the operation. Late period complications were determined from the clinical follow-up and file data of patients.

\section{Statistical analysis}

Statistical analysis was performed using the SPSS for Windows version 15.0 software (SPSS Inc., Chicago, IL, USA). Descriptive data were expressed in mean \pm standard deviation (SD) and number and frequency (\%). The student's t-test was used for a comparison of quantitative variants. Qualitative variants were compared using chi-square tests or the Fisher's exact test, as appropriate. The Pearson correlation analysis was used to examine the relationships between the parameters that conform to the normal distribution. A p-value of $<0,05$ was considered statistically significant.

\section{Results}

This study investigated data from a total of 584 cases. The mean age of the subjects was $69.2 \pm 10.7$ years. There was no statistically significant difference observed between the two study groups in terms of age or gender ( $\mathrm{p}=0.3$ and $\mathrm{p}=0.8$, respectively). There was no significant difference between the two groups in the duration of the bypass operations $(p=0.7)$. In Group $U$, 98.8\% of patients had successful IJV catheterization. For these patients, $94.7 \%$ had right IJV catheterization and $4.1 \%$ had left IJV catheterization. Among the $1.2 \%$ of patients with unsuccessful procedures, right or left femoral vein catherization had been performed.

In Group L, 91.5\% of patients had successful IJV catheterization. Of these patients, $85.2 \%$ had right IJV catheterization and $6.3 \%$ had left IJV catheterization. For the $9.5 \%$ of patients with unsuccessful procedures, right or left femoral vein catheterization had been performed. A comparison of procedure success between the two groups showed that CVC with USG gave a statistically higher rate of success $(\mathrm{p}=0.04)$.

There was no statistically significant difference between the two study groups in platelet counts, INR values, and antiaggregant and anticoagulant drug use in the preoperative period. The activated clotting time (ACT) values measured during cardiopulmonary bypass surgery were not identified to be significantly different in the groups $(\mathrm{p}=0.4)$ (Table 1). 


\section{Cureus}

\begin{tabular}{|c|c|c|c|}
\hline & Group U (n=382) & Group L (n=202) & $p$ value \\
\hline Age (years) & $68.7 \pm 12.5$ & $71.2 \pm 9.8$ & NS \\
\hline Gender (male) & $51.8 \%$ & $52.9 \%$ & NS \\
\hline Duration of operation (minutes) & $186.5 \pm 42.7$ & $198.2 \pm 37.5$ & NS \\
\hline Right internal jugular vein catherization (\%) & $94.7 \%$ & $85.2 \%$ & NS \\
\hline Left internal jugular vein catherization (\%) & $4.1 \%$ & $6.3 \%$ & NS \\
\hline Number of successful procedures (\%) & $98.8 \%$ & $91.5 \%$ & $0.04^{*}$ \\
\hline Preoperative platelet level & $152.360 \pm 42.440$ & $168.430 \pm 41.860$ & NS \\
\hline ACT value during cardiopulmonary bypass & $657 \pm 43$ & $598 \pm 68$ & NS \\
\hline Preoperative INR & $1.12 \pm 0.3$ & $1.08 \pm 0.4$ & NS \\
\hline Preoperative antiaggregant use (\%) & $90.5 \%$ & $89.7 \%$ & NS \\
\hline Preoperative anticoagulant use (\%) & $99.1 \%$ & $97.7 \%$ & NS \\
\hline
\end{tabular}

\section{TABLE 1: Characteristics of patients}

NS: Non-significant, ACT: Activated clotting time, INR: International normalized ratio

$*: p<0.05$

When complications after the procedure are investigated, eight patients in Group U and 18 patients in Group L were observed to have a palpable hematoma. Of those developing a hematoma during postoperative monitoring, 12 patients in Group L and eight patients in Group $\mathrm{U}$ resolved on their own with no late complications developing. There was a statistically significant difference observed in terms of hematoma development in Group $L$ ( $p=0.03)$. One patient underwent jugular vein exploration due to hematoma growth in the postoperative period with vein repair procedure completed.

When groups are assessed in terms of carotid artery puncture, two patients in Group U and 15 patients in Group L had a carotid artery puncture identified. There was a statistically significant difference identified between the two groups $(\mathrm{p}=0.01)$. All patients with a carotid artery puncture were observed not to develop late complications.

With radiological imaging performed during the postoperative monitoring of patients, one patient in Group U was observed to develop pneumothorax of $10 \%$ or less with no hemothorax observed. In Group L, 10 patients were observed to have 10\% or less pneumothorax. There was a statistically significant difference in terms of pneumothorax development. All patients with pneumothorax were monitored. All complications were observed to resolve with conventional treatment without developing late complications (Table 2). 


\section{Cureus}

\begin{tabular}{|c|c|c|c|}
\hline & Group U (n=382) & Group L (n=202) & p-value \\
\hline Palpable hematoma (n) & 8 & 18 & $0.03^{*}$ \\
\hline Carotid artery puncture (n) & 2 & 15 & $0.01^{*}$ \\
\hline Pneumothorax (n) & 0 & 10 & $0,01^{*}$ \\
\hline Catheter malposition (n) & 15 & 13 & NS \\
\hline Hemorrhage requiring surgical intervention (n) & 0 & 3 & $0.05^{*}$ \\
\hline
\end{tabular}

\section{TABLE 2: Complications rate}

NS: Non-significant

$*: p<0.05$

\section{Discussion}

The total complication rate was found to be $6 \%$ in Group U while it was $24 \%$ in Group L. USG guidance revealed an $18 \%$ decrease in the overall complication rate. When the total complication rate was compared, USG guidance was more successful in the Landmark technique and statistically significant ( $\mathrm{p}=0.0001$ ). In the literature, central vein catheterization was shown to have mechanical complication rates of $5 \%-19 \%$, thrombotic complication rates of $2 \%-26 \%$, and infection complication rates of 5\%-26\% [9-11]. Many factors play a role in the development of these complications. Leading factors that affect the success of the procedure are morbid obesity, advanced cachexia, scarring developing at the intervention site linked to previous surgery or radiotherapy, comorbid diseases, and the experience of the clinician performing the procedure [12-15].

Ultrasound use during central vein catheterization provides advanced degree benefits to the clinician performing the procedure for the identification and assessment of the target vein in addition to an observation of the anatomic structures around the vein. This method was shown to increase the procedure's success in intensive care patients and severely reduce the procedure's complications in a variety of publications in the literature [16-17]. The main outcome of this study is that for heart surgery patients with very frequent use of antiaggregant and anticoagulants before and during surgery, USG use during preoperative internal jugular vein catheterization may prevent vital complications.

In our study in patients undergoing coronary bypass surgery, the success of jugular vein catheterization using the landmark method before the operation was identified as $91.5 \%$. In studies, the success of this method varies from $85 \%$ to $99 \%$ [17-19]. In our study, carotid artery puncture with this method was identified as $7 \%$; in the literature, this rate was identified from $3 \%-6 \%$, which appears to be nearly the same as our study data. Additionally, pneumothorax and hemothorax were identified as $5 \%$ and $3 \%$ in our study, which appear to comply with values from other studies [19]. This retrospective analysis observed that the total complication rates fell from $26 \%$ to $6 \%$ when USG guidance is used instead of the landmark technique for catheterization with direct visualization of the IJV.

In our study, central venous catheterization accompanied by ultrasound was observed to have 
mechanical complications close to zero. In two patients, carotid artery puncture was identified. In our hospital, there is no contraindication in the preoperative period for cardiovascular surgery, with all patients undergoing coronary artery bypass surgery using daily $100 \mathrm{mg}$ acetylsalicylic acid, $75 \mathrm{mg}$ clopidogrel, and $6000 \mathrm{IU}$ enoxaparin treatment preoperatively. After central venous catheterization with ultrasound guidance, eight patients were observed to have a palpable hematoma, with all hematomas resolving during monitoring. The cause of hematoma in these patients is considered to be linked to the use of antiaggregant and anticoagulant medication preoperatively. Pneumothorax was not observed.

Malposition is another complication that may be observed during central catheterization. In our study, all patients were assessed in terms of catheter malposition with 15 patients in the USG-guided group and 13 patients in the landmark method group observed to have catheters inserted into the jugular vein located in the opposite subclavian vein or rotating in the vein, with a location outside the right atrium. For all catheters with malposition, no function disruption was observed. From this aspect, the methods were not superior to each other in terms of developing malposition.

Studies in the literature with central vein catheterization using ultrasound guidance and the landmark method compared inexperienced clinicians. All clinician groups using USG were shown to have reduced complication rates independent of experience [19-21].

Complications linked to many causes, such as jugular vein path anomalies, the presence of collapsed veins linked to hypovolemia, and difficulty with jugular vein puncture due to carotid beats in patients with uncontrolled hypertension, may be eliminated with USG. In this way, the need for personal experience, which is at the forefront of the landmark method is reduced by direct visualization. Thus, during the training process, it is possible administrators may perform more reliable central vein catheterization.

In our clinic, standard catheters and unmodified ultrasound devices are used for central vein catheterization. The most important problem for these methods is the cost of obtaining USG devices. However, some studies have proven that in spite of these initial costs, central vein catheterization with USG is more cost-effective [22-23]. From this aspect, when advantages such as lower complication rates, requiring less personal experience, and higher procedure success are considered, it is clear that planning to obtain a USG device for central vein catheterization will be more cost-effective in the long term, especially for newly founded centers.

\section{Conclusions}

As a result, central cannulation is a surgical procedure that must be performed in cardiac surgery. In our study, it was proven that the use of USG for internal jugular vein catheterization of patients receiving intense anticoagulant and antiaggregant treatment and hospitalized for coronary bypass surgery reduced the complication rates by a serious proportion. We suggest that ultrasound-guided central venous catheterization is a safe and effective alternative to the landmark technique.

\section{Additional Information}

\section{Disclosures}

Human subjects: Consent was obtained by all participants in this study. Hitit University Clinical Research Ethical Committee issued approval 2018-13. Animal subjects: All authors have confirmed that this study did not involve animal subjects or tissue. Conflicts of interest: In compliance with the ICMJE uniform disclosure form, all authors declare the following: 
Payment/services info: All authors have declared that no financial support was received from any organization for the submitted work. Financial relationships: All authors have declared that they have no financial relationships at present or within the previous three years with any organizations that might have an interest in the submitted work. Other relationships: All authors have declared that there are no other relationships or activities that could appear to have influenced the submitted work.

\section{References}

1. Frankel A: Temporary access and central venous catheters . Eur J Vasc Endovasc Surg. 2006, 31:417-22. 10.1016/j.ejvs.2005.10.003

2. Marcus HE, Bonkat E, Dagtekin O, et al.: The impact of Trendelenburg position and positive end-expiratory pressure on the internal jugular cross-sectional area. Anesth Analg. 2010, 111:432-436. 10.1213/ANE.0b013e3181e2fe41

3. Augoustides JG, Horak J, Ochroch AE, et al.: A randomized controlled clinical trial of real-time needle-guided ultrasound for internal jugular venous cannulation in a large university anesthesia department. J Cardiothorac Vasc Anesth. 2005, 19:310-315. 10.1053/j.jvca.2005.03.007

4. Legler D, Nugent M: Doppler localization of the internal jugular vein facilitates central venous cannulation. Anesthesiology. 1984, 60:481-482.

5. Bose N, Patel H, Kamat H: Evaluation of ultrasound for central venous access in ICU by an in experienced trainee. Indian J Crit Care Med. 2014, 18:26-32.

6. Balls A, LoVecchio F, Kroeger A, Stapczynski S, Mulrow M, Drachman D: Ultrasound guidance for central venous catheter placement: results from the Central Line Emergency Access Registry Database. Am J Emerg Med. 2010, 28:561-567. 10.1016/j.ajem.2009.02.003

7. Jijeh AM, Shaath G, Kabbani MS, Elbarbary M, Ismail S: Ultrasound guided vascular access in pediatric cardiac critical care. J Saudi Heart Assoc. 2014, 26:199-203.

10.1016/j.jsha.2014.04.003

8. Hoffman T, Du Plessis M, Prekupec MP, Gielecki J, Zurada A, Tubbs RS, Loukas M: Ultrasound-guided central venous catheterization: a review of the relevant anatomy, technique, complications, and anatomical variations. Clin Anat. 2017, 30:237-250. 10.1002/ca.22768

9. McGee DC, Gould MK: Preventing complications of central venous catheterization . N Engl J Med. 2003, 348:1123-1133. 10.1056/NEJMra011883

10. Merrer J, De Jonghe B, Golliot F, et al.: French Catheter Study Group in Intensive Care. Complications of femoral and subclavian venous catheterization in critically ill patients: a randomized control trial. JAMA. 2001, 286:700-707. 10.1001/jama.286.6.700

11. Richards MJ, Edwards JR, Culver DH, Gaynes RP: Nosocomial infections in medical intensive care units in the United States.National Nosocomial Infections Surveillance System. Crit Care Med. 1999, 27:887-892.

12. Sznajder JI, Zveibil FR, Bitterman H, Weiner P, Bursztein S: Central vein catheterization: failure and complication rates by three percutaneous approaches. Arch Intern Med. 1986, 146:259-261. 10.1001/archinte.1986.00360140065007

13. Mansfield PF, Hohn DC, Fornage BD, Gregurich MA, Ota DM: Complication and failures of subclavian-vein catheterization. N Engl J Med. 1994, 331:1735-1738. 10.1056/NEJM199412293312602

14. Randolph AG, Cook DJ, Gonzales CA, Pribble CG: Ultrasound guidance for placement of central venous catheters: a metaanalysis of the literature. Crit Care Med. 1996, 24:2053-2058.

15. Machi J, Takeda J, Kakegawa T: Safe jugular and subclavian venipuncture under ultrasonographic guidance. Am J Surg. 1987, 153:321-323. 10.1016/0002-9610(87)90619-2

16. Malloy DL, McGee WT, Shawker TH, et al.: Ultrasound guidance improves the success rate of internal jugular vein cannulation: a prospective, randomized trial. Chest. 1990, 98:157-160. 10.1378/chest.98.1.157

17. Dennys BG, Uretsky BF, Reddy S: Ultrasound-assisted cannulation of the internal jugular vein a prospective comparison to the external landmark-guided technique. Circulation. 1993, 87:1557-1562. 10.1161/01.CIR.87.5.1557

18. Schwartz AJ, Jobes DR, Greenhow DE, Stephenson LW, Ellison N: Carotid artery puncture with 


\section{Cureus}

internal jugular cannulation using the Seldinger technique: incidence, recognition, treatment, and prevention. Anesthesiology. 1979, 51:160.

19. Gordon AC, Saliken JC, Johns D, Owen R, Gray RR: US-guided puncture of the internal jugular vein complications and anatomic considerations. J Vasc Interv Radiol. 1998, 9:333-338.

20. Gilbert TB, Seneff MG, Becker RB: Facilitation of internal jugular venous cannulation using an audio-guided Doppler ultrasound vascular access device: results from a prospective, dualcenter, randomized, crossover clinical study. Crit Care Med. 1995, 23:60-65.

21. Gualtieri E, Deppe SA, Sipperly ME, Thompson DR: Subclavian venous catheterization: greater success rate for less experienced operators using ultrasound guidance. Crit Care Med. 1995, 23:692-697.

22. Bold RJ, Winchester DJ, Madary AR, Gregurich MA, Mansfield PF: Prospective, randomized trial of Doppler-assisted subclavian vein catheterization. Arch Surg. 1998, 133:1089-1093. 10.1001/archsurg.133.10.1089

23. Calvert N, Hind D, McWilliams R, Davidson A, Beverley CA, Thomas SM: Ultrasound for central venous cannulation: economic evaluation of cost-effectiveness. Anaesthesia. 2004, 59:1116-1120. 10.1111/j.1365-2044.2004.03906.x 